\title{
Analysis of the condition of microbiocenoses and antibiotic resistance on a dairy farm in three-year dynamics
}

\author{
Albina Isaeva ${ }^{1,2, *}$, Anna Krivonogova ${ }^{2}$, Anastasia Chentsova ${ }^{1}, K_{\text {Ksenia }}$ Moiseeva $^{2}$, and \\ Marina Andruysheckina ${ }^{1}$ \\ ${ }^{1}$ Ural State Agrarian University, Department of Infectious and Non-infectious Pathology, 620075 \\ Yekaterinburg, Russia \\ ${ }^{2}$ Federal State Budgetary Scientific Institution «Ural Federal Agrarian Scientific Research Centre, \\ Ural Branch of Russian Academy of Sciences», 620142 Yekaterinburg, Russia
}

\begin{abstract}
The researches studied opportunistic microbiocenoses at different technological sites of a commercial dairy farm in a three-year dynamics. The composition and sensitivity of the opportunistic pathogenic microflora to antibiotics were determined. It was established that the generic and species composition of the core of microbiocenoses was represented by typical opportunistic strains and was characterized by relative constancy at each site. The most common representatives were Enterococcus spp., P. aeruginosa, Proteus spp., S. aureus, E. coli, and C. albicans. A comparative analysis of antibiotic resistance in 2017 and 2020 revealed an upward trend in AMR. At all the examined sites, an increase in the proportion of microorganisms with reduced sensitivity to three or more antibiotics was revealed. An increase in the number of multi-resistant isolates was found in the calve site.
\end{abstract}

\section{Introduction}

The level of antimicrobial resistance (AMR) at livestock facilities has been continuously increasing in recent decades. Despite the measures taken to inhibit the AMR, it is not yet possible to stop or slow down this process [1]. Microorganisms exhibit high adaptive abilities, and the constant use of antibiotics in animal husbandry - including for nontherapeutic purposes - leads to persistent contamination of livestock objects with AMR agents $[2,3]$. On commercial dairy farms, the transmission of AMR and microorganism genes is carried out through several vectors, including contact and contamination of newborn calves with resistant microflora of maternity barns; movement of livestock, distribution with inventory, milk for feeding calves, etc. $[4,5]$. Contamination of farms with AMR agents leads to a decrease in the antibiotic effectiveness, a deterioration in the product quality due to the penetration of microorganisms and resistance genes into it, and in addition, it is dangerous for farm workers, since there is a high risk of replacing opportunistic human microorganisms with resistant isolates circulating in the livestock

\footnotetext{
*Corresponding author: isaeva.05@bk.ru
} 
enterprise [2,6]. In this regard, it is important to monitor the levels of livestock facility contamination with AMR agents and to take timely measures to inhibit resistance.

\section{Materials and methods}

A study of the state of opportunistic microbiocenoses of a commercial dairy farm with the determination of the composition and antibiotic resistance of the opportunistic microflora was conducted for three years. Sampling for microbiological analysis was carried out at all technological sections of the CDF. In the milking livestock departments, the maternity barn, and the calf barn, biomaterial was collected from animals with a coverage of $10 \%$ of the livestock, from randomly selected animals located at different points of the facility. Mandatory points of analysis were swabs from the mucous membranes of the nasal, oral cavity, vagina, wool cover, nipples in cows; nasal, oral cavity, wool cover in calves; swabs from the contact surfaces of equipment, feeders, drinkers, equipment for calf watering, fences, tie devices, surfaces, equipment for livestock caring; samples of bedding, readymade feed mixture, indoor air (total - 218 samples). Microorganisms from the taken material were sown on culture media, cultured, a clean line was isolated, identified, and the sensitivity to antibiotics was analyzed by the disco-diffusion method and the volume displacement method (MUK 4.2.1890-04 Determination of the sensitivity of microorganisms to antibacterial preparations. Guidelines) [7].

The sensitivity of microorganisms to the following antibacterial drugs (ABP) was studied: benzylpenicillin, ampicillin, amoxicillin, meropenem, rifampicin, tetracycline, cefazolin, enrofloxacin, azithromycin, vancomycin, levomycetin. Additionally, individual isolates were analyzed for sensitivity to methicillin, erythromycin, tobramycin, clindamycin, and novobiocin. The main list of antibiotics for tests for antibiotic sensitivity was selected considering the history of antibiotic use in this livestock enterprise over the past two years. The analysis was carried out according to standard microbiological methods. When processing the data, the frequency of occurrence of isolates of opportunistic microorganisms in the samples was evaluated, the total sensitivity of the isolated isolates to each antibiotic was calculated, as well as the total sensitivity of the main pathogenic strains to antibiotics was calculated [8]. When assessing antibiotic sensitivity, only the results of samples with target microorganisms which susceptibility to this antibiotic is known and declared by the manufacturer were considered. Microorganisms that have a natural resistance to a particular preparation were excluded from processing. The results were analyzed using mathematical statistics in the program STATISTICA 10 with the calculation of the average value, standard deviation, and normality testing by the Shapiro-Wilk criterion. The obtained data were used to compile the passport of microbial resistance of the enterprise.

\section{Results}

The results of our studies showed that the generic and specific microflora composition on the commercial dairy farm was mainly represented by typical opportunistic strains and was relatively the same at each technological site. The most pronounced fluctuations in the structure of microbiocenoses were presumably associated with the implementation of sanitary, hygienic, and therapeutic measures. Thus, in the maternity barn and in dairy livestock, a decrease in the proportion of Enterococcus spp. and P. aeruginosa isolates among all isolated ones was noted, while an increase in the proportion of S. aureus was noted (Table 1). In the calf barn in 2020, the share of E. faecium S. aureus increased by more than 2 times compared to 2017. There was a significant decrease in the proportion of 
mold fungi Aspergillius spp., Mucor spp. and Penicillium spp. in the samples taken in the calf barn, which, apparently, is associated with the improvement of the technology of feed preparation and storage at the enterprise. The increase in the proportion of Candida albicans in the three-year period in the examined sites is noteworthy.

Table 1. Structure of the nucleus of opportunistic microbiocenosis at CDF in 2017 and 2020

\begin{tabular}{|c|c|c|c|c|c|c|}
\hline \multirow{2}{*}{ Microorganism } & \multicolumn{2}{|c|}{ Maternity barn } & \multicolumn{2}{|c|}{ Calf barn } & \multicolumn{2}{|c|}{ Dairy livestock barn } \\
\hline & 2017 & 2020 & 2017 & 2020 & 2017 & 2020 \\
\hline $\begin{array}{l}\text { Enterococcus } \\
\text { faecium }\end{array}$ & $23.3 \%$ & $13.3 \%$ & $6.2 \%$ & $14.6 \%$ & $15.3 \%$ & $12.70 \%$ \\
\hline Enterococcus faecalis & $5.3 \%$ & $10.0 \%$ & $7.1 \%$ & $8.5 \%$ & $15.3 \%$ & $9.20 \%$ \\
\hline $\begin{array}{l}\text { Staphylococcus } \\
\text { aureus }\end{array}$ & $3.2 \%$ & $6.40 \%$ & $7.5 \%$ & $22.4 \%$ & $11.5 \%$ & $18.60 \%$ \\
\hline $\begin{array}{l}\text { Staphylococcus } \\
\text { saprophyticus }\end{array}$ & $3.7 \%$ & $0 \%$ & $0 \%$ & $0 \%$ & $0 \%$ & $0 \%$ \\
\hline $\begin{array}{l}\text { Staphylococcus } \\
\text { epidermidis }\end{array}$ & $1.1 \%$ & $2.60 \%$ & $11.1 \%$ & $0.90 \%$ & $0 \%$ & $0 \%$ \\
\hline $\begin{array}{c}\text { Pseudomonas } \\
\text { aeruginosa }\end{array}$ & $16.7 \%$ & $10.8 \%$ & $14.9 \%$ & $13.50 \%$ & $10.2 \%$ & $4.20 \%$ \\
\hline Enterobacter spp. & $5.9 \%$ & $6.6 \%$ & $11.0 \%$ & $14.3 \%$ & $7.0 \%$ & $11.10 \%$ \\
\hline Echerichia coli & $4.0 \%$ & $18.9 \%$ & $5.5 \%$ & $7.1 \%$ & $7.5 \%$ & $7.53 \%$ \\
\hline Proteus spp. & $11.6 \%$ & $7.20 \%$ & $0.0 \%$ & $4.20 \%$ & $12.0 \%$ & $7.02 \%$ \\
\hline Bacillus spp. & $\begin{array}{c}\text { less than } \\
0.5 \% \\
\end{array}$ & $1.10 \%$ & $8.20 \%$ & $5.5 \%$ & $1.25 \%$ & $4.01 \%$ \\
\hline Candida albicans & $8.5 \%$ & $10.2 \%$ & $2.5 \%$ & $3.80 \%$ & $7.7 \%$ & $12.40 \%$ \\
\hline Mucor spp. & $5.7 \%$ & $2.40 \%$ & $11.0 \%$ & $1.60 \%$ & $7.6 \%$ & $3.40 \%$ \\
\hline Fusarium & $4.4 \%$ & $1.80 \%$ & - & - & $3.8 \%$ & $4.40 \%$ \\
\hline Penicillium & $2.0 \%$ & $3.50 \%$ & $5.6 \%$ & $2.10 \%$ & - & - \\
\hline Aspergillus spp. & $3.1 \%$ & $3.50 \%$ & $7.7 \%$ & $\begin{array}{c}\text { less than } \\
0.5 \%\end{array}$ & - & - \\
\hline Other, total & $1.50 \%$ & $1.70 \%$ & $1.4 \%$ & $1.50 \%$ & $0.8 \%$ & $5.44 \%$ \\
\hline
\end{tabular}

The analysis of the antibiotic sensitivity of the isolates showed a general trend towards an increase in the proportion of resistant microorganisms. In the maternity barn, the number of isolates not sensitive to amoxicillin, clindamycin, levomycetin, and azithromycin increased most significantly (Figure 1). In addition, in 2020, isolates resistant to rifampicin were detected (their share of all isolated was $4 \%$ ), while in 2017 , no microorganisms with such an AMR profile were detected. Due to the fact that rifampicin has antituberculotic activity, it is highly undesirable to use it for the treatment of infections not associated with mycobacteria. The acquisition of rifampicin resistance by facultative pathogens may adversely affect both the effectiveness of rifampicin and the spread of resistance genes.

There was a slight decrease in the level of resistance to ampicillin (by $3 \%$ ), which could be associated both with the refusal to use this antibiotic, and with a decrease in the number of susceptible microorganisms in the microbiocenosis.

At the technological site of calves in 2020, a decrease in the level of resistance to meropenem (by 13\%), tetracycline (by 14\%), enrofloxacin and azithromycin was revealed by more than 2 times compared to the results of 2017. At the same time, the proportion of isolates resistant to amoxicillin increased by 2.5 times. 
High levels of resistance to ampicillin, amoxicillin, tetracycline, and cefazolin were detected in both dairy livestock barns (Figure 2). However, in 2020, there was a significant decrease in the proportion of ampicillin-and levomycetin-resistant isolates. The number of isolates resistant to clindamycin, on the contrary, increased to $16 \%$. Presumably, this is due to the replacement of drugs for antibiotic therapy.

\section{Dynamics of the number of resistant isolates in the maternity barn of the CDF in 2017-2020}

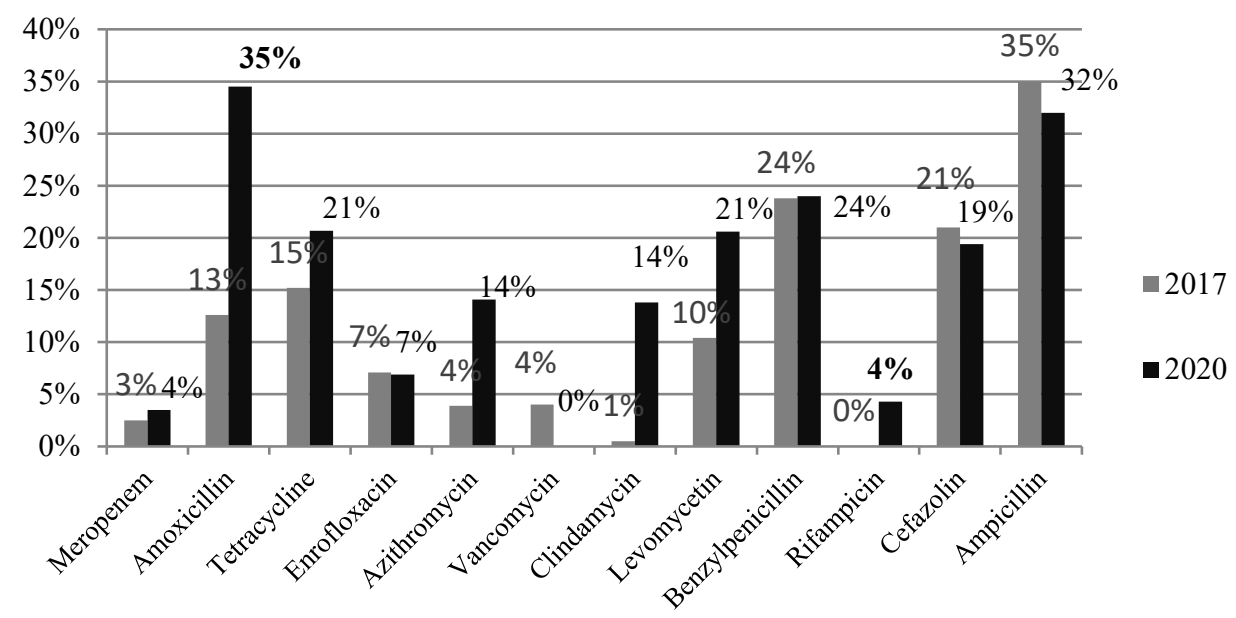

Fig. 1. Dynamics of the number of resistant isolates in the maternity barn of the examined dairy farm in 2017-2020.

\section{Dynamics of the number of resistant isolates in dairy livestock barns of the CDF in 2017-2020}

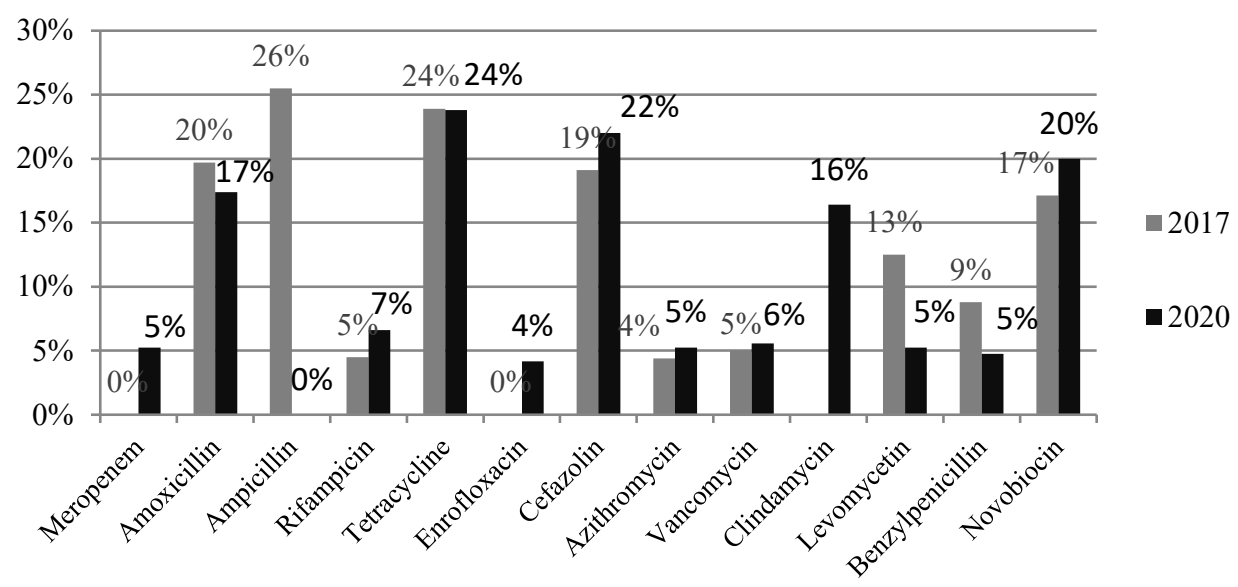

Fig. 2. Dynamics of the number of resistant isolates in two dairy livestock barns of the CDF in 20172020.

The analysis of the multiresistance characteristics showed that in the maternity barn, the proportion of isolates resistant to 2 or more classes of ABP (Mr) decreased by $10 \%$ over 
three years, in the areas of dairy livestock - decreased slightly (by $2 \%$ ), and in the calf sites increased by $3 \%$ (Figure 3 ).

\section{Dynamics of the number of multiresistant isolates from all with} AMR at the CDF in 2017-2020

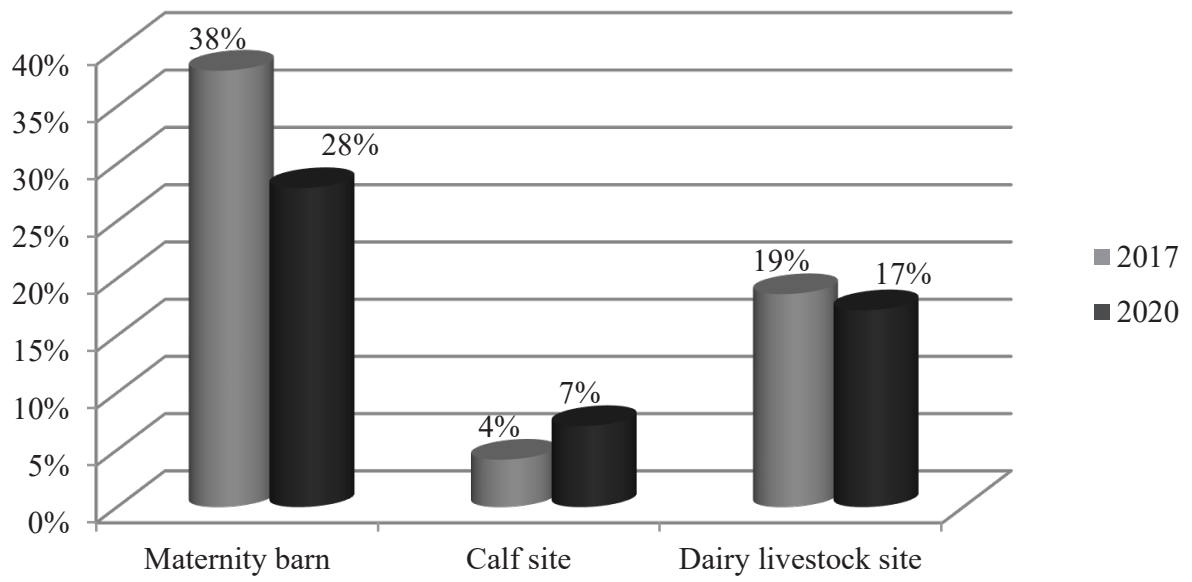

Fig. 3. Change in the number of isolates resistant to 2 or more classes of ABP in the technological sites of the CDF in 2017-2020.

At the same time, there was a tendency to increase the number of isolates with weak sensitivity to 3 or more antibiotics, but not yet showing signs of multi-resistance (Figure 4). These isolates are in a borderline state with a high risk of complete loss of susceptibility to several ABPs and make up a significant amount of all isolated isolates. The existence of such a group can significantly worsen the AMR indicators at the enterprise in the future.

\section{Dynamics of isolates with reduced sensitivity to several antibiotics at the CDF in 2017-2020}

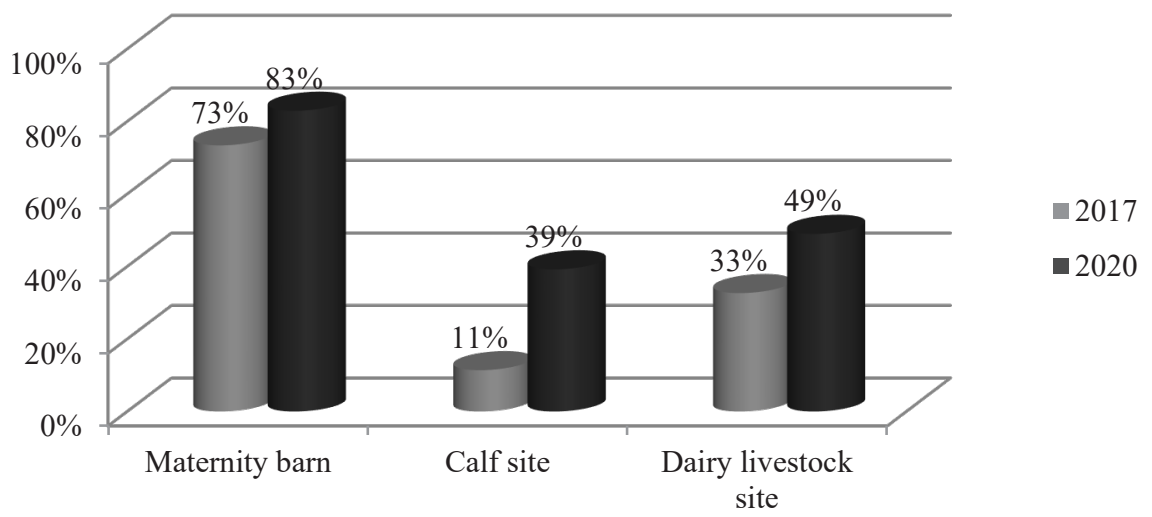

Fig. 4. Dynamics of the number of isolates with reduced sensitivity to three or more antibiotics in 2017-2020. 


\section{Conclusions}

The conducted studies have shown that on the commercial dairy farm there is a tendency to a gradual increase in AMR, mediated through the actual increase in the number of resistant isolates, as well as through the increase in the number of microorganisms with reduced sensitivity to ABP. In addition, a high level of multiple resistance was observed in all the examined CDF sites, ranging from $28-38 \%$ in the maternity barn to $4-7 \%$ in the calf barn. The increase in the proportion of microorganisms that lose their sensitivity to several antibiotics at once over a three-year time period was observed at all sites.

\section{Acknowledgment}

The research is executed at the expense of a grant of the Russian scientific fund (project No. 18-16-00040).

\section{References}

1. L.C. Ventola, Pharmacy and Therapeutics 40, 1-3 (2015)

2. M.F. Khan, EC Microbiology 5, 36-39 (2017)

3. E.L. Ilchenko, Yu.A. Smirnova, Study of the resistance of staphylococcus class bacteria to broad-spectrum antibiotics, In the collection: New tasks of modern medicine Proceedings of the V International Scientific Conference, 28-35 (2018)

4. C.J.H. von Wintersdorff, J. Penders, J.M. van Niekerk, N.D. Mills, S. Majumder, L.B. van Alphen, P.H.M. Savelkoul, P.F.G. Wolffs, Frontiers in Microbiology 7, 21 (2016)

5. A.S. Krivonogova, A.S. Romanova, A.G. Isaeva, K.V. Moiseeva, V.S. Pashtetskiy, Proceedings of the Kuban State Agrarian University 84, 246-254 (2020)

6. Sun, Jian \& Huang, Ting \& Chen, Chong \& Cao, Ting-Ting \& Cheng, Ke \& Liao, Xiao-Ping \& Liu, Ya-Hong, Scientific Reports, 7 (2017) 10.1038/s41598-017-04672-y

7. Clinical recommendations. Determination of the susceptibility of microorganisms to antimicrobials (Clinical guidelines. Determination of the sensitivity of microorganisms to antimicrobial drugs), 2015.2 URL: http://www.antibiotic.ru/minzdrav/files/docs/clrec-dsma2018.pdf (date of access 18.08.2020).

8. A.S. Krivonogova, I.M. Donnik, A.G. Isaeva, K.V. Moiseeva, Agrarian Bulletin of the Urals 9(200), 42-47 (2020) 